Corresponding author: pevsner@kennedykrieger.org

(C) 2020 Osei-Owusu et al. This article is distributed under the terms of the Creative Commons Attribution-NonCommercial License, which permits reuse and redistribution, except for commercial purposes, provided that the original author and source are credited.

Ontology terms: attention deficit hyperactivity disorder; autism; intellectual disability, severe; language impairment

Published by Cold Spring Harbor Laboratory Press

doi:10.1101/mcs.a005884

\section{Characterization of an unbalanced translocation causing 3q28qter duplication and 10q26.2qter deletion in a patient with global developmental delay and self-injury}

\author{
Ikeoluwa A. Osei-Owusu, ${ }^{1,2}$ Alexis L. Norris, ${ }^{2,8}$ Anya T. Joynt, ${ }^{1}$ Jeremy Thorpe ${ }^{2,3}$ \\ Soonweng Cho, ${ }^{2,4,9}$ Elaine Tierney, ${ }^{4,5}$ Jonathan Schmidt, ${ }^{4,6}$ Louis Hagopian, ${ }^{4,6}$ \\ Jacqueline Harris, ${ }^{2,7}$ and Jonathan Pevsner ${ }^{1,2,3,4}$

\begin{abstract}
${ }^{1}$ Program in Human Genetics, Johns Hopkins School of Medicine, Baltimore, Maryland 21205, USA; ${ }^{2}$ Department of Neurology, Kennedy Krieger Institute, Baltimore, Maryland 21205, USA; ${ }^{3}$ Program in Biochemistry, Cellular and Molecular Biology, ${ }^{4}$ Department of Psychiatry and Behavioral Sciences, Johns Hopkins School of Medicine, Baltimore, Maryland 21205, USA; ${ }^{5}$ Department of Psychiatry, ${ }^{6}$ Department of Behavioral Psychology, Kennedy Krieger Institute, Baltimore, Maryland 21205, USA; ${ }^{7}$ Department of Neurology, Johns Hopkins School of Medicine, Baltimore, Maryland 21205, USA
\end{abstract}

\begin{abstract}
Chromosomal structural variation can cause severe neurodevelopmental and neuropsychiatric phenotypes. Here we present a nonverbal female adolescent with severe stereotypic movement disorder with severe problem behavior (e.g., self-injurious behavior, aggression, and disruptive and destructive behaviors), autism spectrum disorder, severe intellectual disability, attention deficit hyperactivity disorder, and global developmental delay. Previous cytogenetic analysis revealed balanced translocations present in the patient's apparently normal mother. We hypothesized the presence of unbalanced translocations in the patient due to maternal history of spontaneous abortions. Whole-genome sequencing and whole-genome optical mapping, complementary next-generation genomic technologies capable of the accurate and robust detection of structural variants, identified $t(3 ; 10)$, $\mathrm{t}(10 ; 14)$, and $\mathrm{t}(3 ; 14)$ three-way balanced translocations in the mother and der(10)t(3;14;10) and $\operatorname{der}(14) \mathrm{t}(3 ; 14 ; 10)$ translocations in the patient. Instead of a $\mathrm{t}(3 ; 10)$, she inherited a normal maternal copy of Chromosome 3, resulting in an unbalanced state of a 3q28qter duplication and 10q26.2qter deletion. Copy-imbalanced genes in one or both of these regions, such as DLG1, DOCK1, and EBF3, may contribute to the patient's phenotype that spans neurodevelopmental, musculoskeletal, and psychiatric domains, with the possible contribution of a maternally inherited $15 q 13.2 q 13.3$ deletion.
\end{abstract}

[Supplemental material is available for this article.]

\section{INTRODUCTION}

Structural chromosomal abnormalities, such as translocations and copy-number variants (CNVs), collectively play a significant role in susceptibility to various neurodevelopmental

\footnotetext{
${ }^{8}$ Present address: Center for Veterinary Medicine, Food and Drug Administration, Rockville, Maryland 20855, USA

${ }^{9}$ Present address: Arcus Biosciences, Hayward, California 94545, USA
} 
and neuropsychiatric disorders, including epilepsy, autism spectrum disorder (ASD), intellectual disability (ID), and schizophrenia (Vassos et al. 2010; Talkowski et al. 2012). Chromosomal translocations-balanced or unbalanced-may result in disruption of genomic loci at translocation breakpoints. Although balanced translocations often produce no phenotypic effects, the segregation of an unbalanced form of a translocation may occur in a child of an apparently normal individual as a result of gain and loss of genomic material (Baptista et al. 2005; Weckselblatt et al. 2015). Identifying disrupted genes is important for understanding the phenotypic consequences of translocations. The use of limited-resolution and low-efficiency traditional techniques, such as G-banded karyotyping, fluorescence in situ hybridization (FISH), and microsatellite marker genotyping, limits the ability to detect clinically relevant structural variants (SVs), especially as tools in genetic counseling for specific neurodevelopmental disorders (Vassos et al. 2010; Lin et al. 2016). Instead, nextgeneration sequencing (NGS) approaches facilitate localization of chromosomal breakpoints to nucleotide-level precision and reliably delineate copy-number changes (Ordulu et al. 2016). In addition, whole-genome optical mapping is a sensitive tool for detecting large genomic variants and resolving genomic regions with duplications or higher-order repeats typically difficult for short-read NGS technology (Barseghyan et al. 2017). These technologies enable the characterization of complex chromosomal rearrangements as in the case of our patient, improving our understanding of their clinical relevance.

\section{RESULTS}

\section{Clinical Presentation}

Our patient was a nonverbal 16-yr-old Caucasian female hospitalized for the assessment and treatment of severe problem behavior. Her diagnoses included stereotypic movement disorder with self-injurious behavior (SIB), global developmental delay, ASD, severe ID, attention deficit hyperactivity disorder (ADHD), and mixed receptive-expressive language disorder (Table 1). Physical exam was significant for short stature, large head with a long, slightly asymmetrical jaw and large forehead, large and prominent ears, downturned corners of the mouth, downslanting palpebral fissures, beaked nose, diffuse hypotonia, spastic dysarthria, scoliosis, limited range of motion at the feet and ankles, and a wide-based gait. Her problem behaviors included severe SIB (hitting head with open and closed fist, head banging), aggression (grabbing others, hair pulling, hitting, punching, kicking, scratching), disruptive and destructive behaviors (screaming, breaking objects, knocking furniture, throwing items), inappropriate sexual behaviors (placing hands in pants, masturbating), disrobing (attempting to remove clothing in public places), mouthing objects (placing inedible objects in mouth), spitting (expelling food or fluid past the plane of the lips), noncompliance (dropping and/or not following adult directions within $10 \mathrm{sec}$ ), severe sleep dysregulation, and food refusal. Her aggression had increased with age and was further magnified with the onset of puberty. She had undergone multiple medication trials but the medications reportedly increased her irritability and worsened her problem behavior. Because of the severity and risk of injury to herself and others from SIB and aggression, these behaviors were primarily targeted for intervention by first assessing the environmental variables evoking and maintaining the behavior by conducting a functional behavioral assessment. To determine her level of adaptive functioning, the patient's scores on the Vineland Adaptive Behavior Scales were also assessed.

\section{Behavioral Studies}

\section{Functional Behavioral Assessment}

To collect information to construct operational definitions for each form of problem behavior, trained clinicians conducted interviews with parents and school personnel and directly 
Table 1. Clinical characteristics of the patient and their relevance to the $10 \mathrm{q} 26$ deletion and $3 \mathrm{q} 29$ duplication syndromes

\begin{tabular}{|c|c|c|c|c|c|}
\hline Clinical feature & $\mathrm{HPO}^{\mathrm{a}}$ & $\begin{array}{l}\text { Previous } \\
10 q \text { cases }\end{array}$ & References ( $10 q$ cases) & $\begin{array}{l}\text { Previous } \\
3 q \text { cases }\end{array}$ & $\begin{array}{l}\text { References ( } 3 q \\
\text { cases) }\end{array}$ \\
\hline ID, severe & HP:0010864 & $x$ & Courtens et al. 2006 & $x$ & $\begin{array}{l}\text { Fernández-Jaén } \\
\text { et al. } 2014\end{array}$ \\
\hline ASD & HP:0000717 & $x$ & Yatsenko et al. 2009 & $x$ & Pollak et al. 2020 \\
\hline ADHD & HP:0007018 & $x$ & $\begin{array}{l}\text { Courtens et al. 2006; } \\
\text { Yatsenko et al. } 2009\end{array}$ & $x$ & Pollak et al. 2020 \\
\hline Language impairment & HP:0002463 & $x$ & Courtens et al. 2006) & $x$ & Pollak et al. 2020 \\
\hline Motor delay & HP:0001270 & $x$ & Courtens et al. 2006 & $x$ & Pollak et al. 2020 \\
\hline Stereotypy (hand and eye) & HP:0000733 & $x$ & Yatsenko et al. 2009 & $x$ & $\begin{array}{l}\text { Fernández-Jaén } \\
\text { et al. } 2014\end{array}$ \\
\hline Mood changes (dysthymia/anhedonia) & HP:0001575 & $x$ & Courtens et al. 2006 & $x$ & Pollak et al. 2020 \\
\hline Self-injurious behavior & HP:0100716 & $x$ & Courtens et al. 2006 & & \\
\hline $\begin{array}{l}\text { Abnormal aggressive, impulsive or } \\
\text { violent behavior }\end{array}$ & HP:0006919 & $x$ & Courtens et al. 2006 & $x$ & Pollak et al. 2020 \\
\hline Inappropriate sexual behavior & HP:0008768 & $x$ & Courtens et al. 2006) & & \\
\hline Agitation & HP:0000713 & $x$ & Courtens et al. 2006 & & \\
\hline Sleep disturbance & HP:0002360 & $x$ & Courtens et al. 2006 & & \\
\hline Generalized hypotonia & HP:0001290 & $x$ & $\begin{array}{l}\text { Courtens et al. 2006; } \\
\text { Miller et al. 2009b }\end{array}$ & $x$ & Pollak et al. 2020 \\
\hline Gait ataxia & HP:0002066 & $x$ & Miller et al. 2009b & & \\
\hline Macrocephaly $(z=2.14)$ & HP:0000256 & & & $x^{a}$ & Ballif et al. 2008 \\
\hline Short stature $(z=-2.99)$ & HP:0004322 & $x$ & Yatsenko et al. 2009 & & \\
\hline Strabismus & HP:0000565 & $x$ & $\begin{array}{l}\text { Courtens et al. 2006; } \\
\text { Miller et al. 2009b; } \\
\text { Yatsenko et al. } 2009\end{array}$ & & \\
\hline Scoliosis & HP:0002650 & $x$ & Courtens et al. 2006 & $x$ & $\begin{array}{l}\text { Battaglia et al. } \\
2006^{\mathrm{b}}\end{array}$ \\
\hline Talipes equinovarus & HP:0001776 & & & $x$ & $\begin{array}{l}\text { Lawrence et al. } \\
2017\end{array}$ \\
\hline $\begin{array}{l}\text { Specific craniofacial dysmorphisms } \\
\text { (downslanted palpebral fissures; } \\
\text { beaked nose; protruding ears; broad } \\
\text { forehead) }\end{array}$ & $\begin{array}{l}\text { HP:0000494 } \\
\text { HP:0000366 } \\
\text { HP:0000411 } \\
\text { HP:0000337 }\end{array}$ & $x$ & $\begin{array}{l}\text { Courtens et al. 2006; } \\
\text { Yatsenko et al. } 2009\end{array}$ & $x$ & Lisi et al. 2008 \\
\hline
\end{tabular}

Because our patient has a 10q26.2qter deletion we focus the cited literature on patient(s) with overlapping deletions. Some 10q26 deletion syndrome literature spanning 10q26.11qter, 10q26.12qter, 10q26.13qter, and 10q26.3qter also have overlapping features with our patient (OMIM \#609625). Human Phenotype Ontology data are from the November 2019 release (https://hpo.jax.org/app/).

${ }^{a}$ Reported in a few cases, although most display microcephaly.

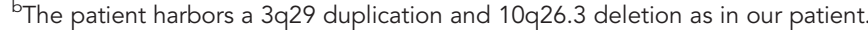

(HPO) Human Phenotype Ontology, (ID) intellectual disability, (ASD) autism spectrum disorder, (ADHD) attention deficit hyperactivity disorder.

observed the patient. We performed a functional analysis of the patient's SIB and aggression to identify the controlling antecedent and consequent events that occasion and maintain these behaviors. Although the methodology is standardized, the assessment procedures are individualized based on the participant's target behaviors from their reported history. This assessment involves conducting a controlled analysis of the contingency classes by systematically manipulating the antecedents and consequences across four conditions (ignore, verbal attention, demand, and a control condition). Each condition is designed to simulate conditions the patient typically encountered. A series of 10-min sessions were conducted 
where conditions were alternated systematically. Behavioral data were collected by trained observers using computer software (Bullock et al. 2017), and findings were interpreted based on validated criteria (Hagopian et al. 1997). Specifically, behavioral data were graphically depicted and interpreted using established conventions. The patterns and rates of problem behavior for each condition were compared to those in the control condition to identify the operant reinforcing function(s) of SIB and aggression. Using this same framework, subsequent analyses were conducted to more specifically isolate the variables affecting the occurrence of the patient's SIB and aggression related to the delivery of physical attention (hand holding and removal and blocking the occurrence of SIB). Results of the initial functional analysis were inconclusive because of variable and low levels of behaviors across conditions; however, subsequent analysis indicated that aggression was likely maintained by access to physical attention. Specifically, during test conditions when physical attention was provided contingent on the occurrence of aggression, this behavior occurred at a significantly higher rate (mean $[\mathrm{M}]=1.2$ aggressions per minute) relative to control conditions when physical attention was provided freely ( $M=0.3$ aggressions per minute). Results regarding the function of SIB under these conditions were inconclusive.

\section{Vineland Adaptive Behavior Scales}

Measures of adaptive behavior were obtained from the patient's parents via the Vineland Adaptive Behavior Scales-Second edition (Vineland-II) Parent/Caregiver Rating Form (Sparrow et al. 2005). The Vineland-Il measures adaptive behavior across domains of Communication (i.e., expressive, receptive, and written language skills), Daily Living (i.e., personal, domestic, and community-based skills), Socialization (interpersonal relationships, play and leisure, and coping skills), and Motor Skills (i.e., fine and gross motor skills). For each domain, raw scores were converted into standard scores $(M=100, S D=15)$, and a comprehensive adaptive behavior composite score $(M=100, S D=15)$. All standard scores were categorized as one of five levels of adaptive functioning: low, moderately low, adequate, moderately high, and high. All of the patient's scores on the Communication (Standard Score $[S S]=40$ ), Daily Living Skills (SS $=28)$, and Socialization domains (SS $=40$ ), as well as the Adaptive Behavior Composite (SS = 33), on the Vineland-II (Sparrow et al. 2005) were categorized in the low range of adaptive functioning. These scores were commensurate with her prior diagnoses.

\section{Treatment Outcomes}

Although SIB was infrequent in our analog functional analysis sessions, during the first couple of weeks on the hospital unit the patient engaged in elevated rates of all forms of problem behavior, particularly SIB. After this initial period, she engaged in low but variable rates of SIB throughout the admission; however, SIB often occurred in large bouts with high intensity. A combined behavioral and pharmacological treatment was developed to target the occurrence of the patient's SIB, aggression, and disruption. The final behavioral treatment consisted of multiple components, including systematic arrangement of structured activities (independent or interactive leisure, academics, and physical/sensory activities), planned ignoring for problem behavior, functional communication for reinforcers (attention, preferred toys or food), signaled availability of reinforcers (attention, preferred toys or food), specific prompting procedures, differential reinforcement for compliance with demands via a token economy system, contingent access to preferred items, and contingent blocking of SIB if the behavior occurred five times or more within $1 \mathrm{~min}$. The final pharmacological treatment consisted of daily total doses of doxepin $1.5 \mathrm{mg}$, guanfacine extended release $7 \mathrm{mg}$, and sertraline $100 \mathrm{mg}$. During the initial baseline period, the patient engaged in an average of 291.7 occurrences of self-injurious, aggressive, and disruptive behaviors per day, whereas with the 
combined behavioral and pharmacological treatment she engaged in an average of 44.8 occurrences of self-injurious, aggressive, and disruptive behaviors per day. However, most of the behaviors that were still occurring were less intensive and easily managed. Of most importance due to severity, the patient engaged in SIB only 2 out of 15 days during treatment, with an average of 0.6 occurrences of SIB per day. Additionally, the patient only engaged in an average of 1.8 occurrences of forceful aggression per day (not including grabbing another person's body).

\section{Genomic Analyses}

Prior clinical cytogenetics analysis using karyotyping had reported a complex chromosomal rearrangement. This consisted of balanced translocations involving Chromosomes 3, 10, and 14 in the mother: 46,XX,t(3;14;10)(q27;q22;q26.1).ish $t(3 ; 14 ; 10)(D 3 S 4560-, D 10 S 2490+;$ D14S308-,D3S4560+;D10S2490-,D14S308+). The mother had at least five prior spontaneous abortions, consistent with her having unbalanced translocations in her progeny.

To clarify the nature of SVs in this family, we subjected DNA from the patient and parents to whole-genome sequencing (WGS) analyses. The Manta SV caller confirmed three interchromosomal translocations of $\mathrm{t}(3 ; 10)(\mathrm{q} 28 ; \mathrm{q} 26.2), \mathrm{t}(10 ; 14)(\mathrm{q} 26.2 ; \mathrm{q} 22.3)$, and $\mathrm{t}(3 ; 14)(\mathrm{q} 28$; $\mathrm{q} 22.3)$ in the mother, consistent with the $t(3 ; 14 ; 10)$ rearrangement observed by conventional cytogenetics. The patient harbored the $\operatorname{der}(10) t(3 ; 14 ; 10)$ and $\operatorname{der}(14) t(3 ; 14 ; 10)$ translocations, with no evidence of $t(3 ; 10)(q 28 ; q 26.2)$, but instead inherited the mother's intact copy of Chromosome 3 (Fig. 1). These unbalanced translocations resulted in two segmental aneuploidies: (1) an 8.9-Mb 3q28qter duplication containing 59 protein-coding RefSeq genes (Table 2; Supplemental Table 1) and (2) a 7.2-Mb 10q26.2qter deletion encompassing 41 protein-coding RefSeq genes (Table 2; Supplemental Table 2). The der(10)t(3;14;10) and der(14)t(3;14;10) breakpoints were resolved to base pair level (an advantage of the high-resolution WGS technology). Breakpoints were confirmed in the patient (Fig. 2) as well as the corresponding positions in the mother using the Integrative Genomics Viewer (IGV) (Supplemental Figs. 1-3). We validated these breakpoints by Sanger sequencing of
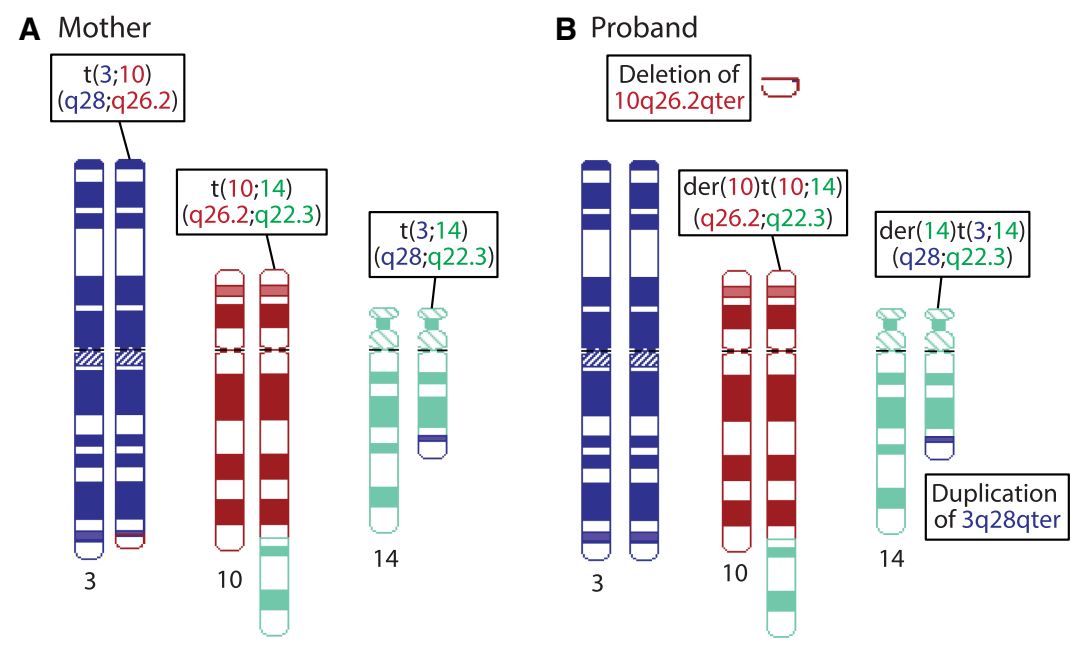

Figure 1. Ideograms representing a model of balanced and unbalanced translocations in the mother and patient. (A) Balanced three-way translocation in the mother. A portion of $\mathrm{Chr} 3$ was translocated to $\mathrm{Chr} 14$, as well as $\mathrm{Chr} 14$ to $\mathrm{Chr} 10$ and Chr 10 to $\mathrm{Chr} 3$. (B) Unbalanced translocation in the patient. The patient inherited the $\mathrm{Chr} 3$ to $\mathrm{Chr} 14$ and Chr 14 to $\mathrm{Chr} 10$ translocations but not the $\mathrm{Chr} 10$ to $\mathrm{Chr} 3$ translocation, instead inheriting two intact copies of $\mathrm{Chr} 3$, one from each parent. This resulted in a de novo 10q26.2qter deletion and 3q28qter duplication. 
Table 2. Summary of detected likely impactful copy-number variants

\begin{tabular}{|c|c|c|c|c|}
\hline & Array genomic position ${ }^{a}$ & WGS genomic position ${ }^{b}$ & Variant type & Classification \\
\hline 3q28qter & $\begin{array}{l}\operatorname{arr}[\text { GRCh37] 3q28q29(189233607_ } \\
\text { 197882598)×3 }\end{array}$ & $\begin{array}{l}\text { Chr 3(GRCh37): } \\
189,132,021-198,022,430\end{array}$ & Duplication & Pathogenic \\
\hline 10q26.2qter & $\begin{array}{l}\operatorname{arr}[\text { GRCh37] 10q26.2q26.3 } \\
\quad\left(128771757 \_135370795\right) \times 1\end{array}$ & Chr 10(GRCh37):128,338,371-135,534,747 & Deletion & Pathogenic \\
\hline $15 q 13.2 q 13.3$ & $\begin{array}{r}\operatorname{arr}[\text { GRCh37] 15q13.2q13.3 } \\
\quad\left(31115047 \_32418279\right) \times l\end{array}$ & Chr 15(GRCh37):30,906,059-32,427,157c & Deletion & Uncertain significance ${ }^{d}$ \\
\hline
\end{tabular}

${ }^{\mathrm{a}}$ Genomic position as provided by the clinical array-based comparative genomic hybridization (aCGH) and single-

nucleotide polymorphism (SNP) genotype analysis report using ISCN, an accepted HGVS nomenclature.

${ }^{\mathrm{b}}$ Genomic position as determined by WGS analysis.

${ }^{\mathrm{c} B r e a k p o i n t s ~ w e r e ~ e s t i m a t e d ~ f r o m ~ W G S ~ a n d ~ w h o l e-o p t i c a l ~ m a p p i n g ~ C N V ~ a n a l y s e s . ~}$

${ }^{\mathrm{d}}$ This classification differs from the clinical aCGH and SNP report of pathogenic with the recommendation to test parental

samples to determine if the variant is de novo or inherited.

polymerase chain reaction (PCR) products and confirmed the CNVs with coverage data bioinformatics analyses (Supplemental Figure 4; see Supplemental Text 1).

Assessing these chromosomal changes using an orthogonal method, we performed next-generation whole-genome optical mapping using the Bionano Genomics Saphyr system. Two optical maps were generated from single DNA molecules labeled at specific restriction sites and used for de novo genome assembly. These maps provided long-range structural information that confirmed the three-way balanced translocations of $t(3 ; 10)$, $t(10 ; 14)$, and $t(3 ; 14)$ in the mother (Fig. 3A), with only the latter two translocations occurring in the patient (Fig. 3B; Supplemental Fig. 5). A large 3q28qter duplication (Supplemental Fig. 6) and 10q26.2qter deletion (Supplemental Fig. 7) occurred in the patient but not her mother. Results were consistent with those obtained from Illumina-based WGS.

To validate the results of our sequencing and genome mapping analyses, we used clinical whole-genome array-based comparative genomic hybridization (aCGH) and single-nucleotide polymorphism (SNP) genotype analysis on a DNA sample from the patient only. Three pathogenic CNVs were reported: (1) 8.6-Mb 3q28q29 duplication, (2) 6.6-Mb 10q26.2q26.3 deletion, and (3) 1.3-Mb 15q13.2q13.3 deletion. The former two findings were consistent with the deletion and duplication resulting from the unbalanced translocation in the patient as described above. Our analysis of WGS (data not shown) and optical genome mapping data (Fig. 3; Supplemental Fig. 8) showed that the 15q deletion event was maternally inherited, and therefore of uncertain clinical significance. Using identity by state (IBS) analyses, we confirmed the maternal origin of the 15q (Supplemental Fig. 9A), 10q (Supplemental Fig. 9B; Supplemental text 2), and 3q (Supplemental Fig. 10; Supplemental Text 2) CNVs.

\section{DISCUSSION}

We report complex germline balanced translocations present in an apparently normal adult female whose child harbors a de novo terminal duplication of $3 q 28$ and a terminal deletion of $10 q 26.2$ as a result of incomplete inheritance of the translocated chromosomes. The genomic rearrangements were detected using WGS and whole-genome optical mapping of the mother/father/patient trio. We validated the results using Sanger sequencing of PCR-amplified genomic DNA, IBS analysis of genotypes, and clinical array CGH. Our results were consistent with terminal (rather than interstitial) duplication on $3 q$ and deletion on 10q. Terminal chromosomal regions can be difficult to resolve. In the case of the $10 \mathrm{q}$ deletion in the 


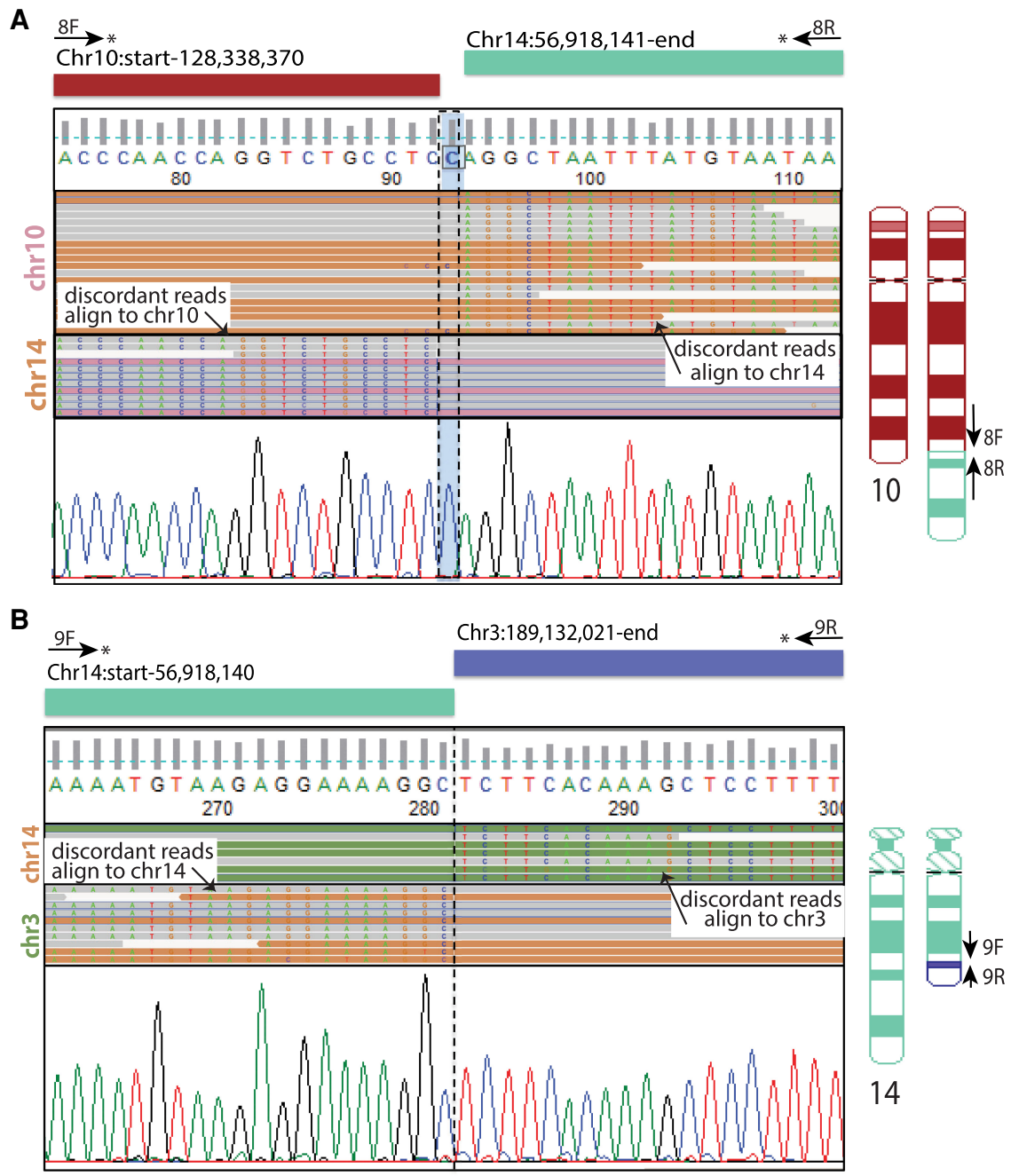

Figure 2. Sequence read evidence at translocation breakpoints in the patient. Reads in BAM files were visualized using the Integrative Genomics Viewer (IGV). Sanger traces were visualized using Finch TV. (A) Representing the der(10)t(3;14;10) breakpoint, an IGV view of Chr 10 and Chr 14 show discordant reads that align to Chr 14 (shaded orange) and Chr 10 (shaded pink), respectively. This is embedded within Sanger sequence traces of this region. PCR primers ( $8 \mathrm{~F}$ and $8 \mathrm{R})$ were used to generate a fragment that spanned the translocation breakpoint between Chromosomes 10 and 14 in the patient. A cytidine residue (shaded in blue column) occurred at a position corresponding to the end of the Chr $10(\mathrm{Chr} 10: 128,338,370)$ and beginning of Chr 14 (Chr 14:56,918,141) sequence. (B) Representing the der(14)t(3;14;10) breakpoint, an IGV view of Chr 14 and Chr 3 show discordant reads that aligned to Chr 3 (shaded green) and Chr 14 (shaded orange), respectively. This is embedded within Sanger sequence traces of this region showing the breakpoint at Chr $14: 56,918,140$ and Chr $3: 189,132,021$. PCR primers (9F and 9R) were used to generate a fragment that spanned the translocation breakpoint between $\mathrm{Chr} 14$ and $\mathrm{Chr} 3$ in the patient. *Arrows indicate primer positions schematically.

patient, we find that the deletion unambiguously extended at least to position Chr 10:135,436,070 (GRCh37; $98 \mathrm{~kb}$ from the annotated end of the chromosome). Starting at the next base position, the region has extensive segmental duplications and distal reads also mapped to canonical telomeric repeats.

The duplicated 3q28qter region overlaps the recently described Chromosome 3q29 duplication syndrome region (Online Mendelian Inheritance in Man [OMIM] \#611936). 
A Structural variants detected in the mother using optical mapping

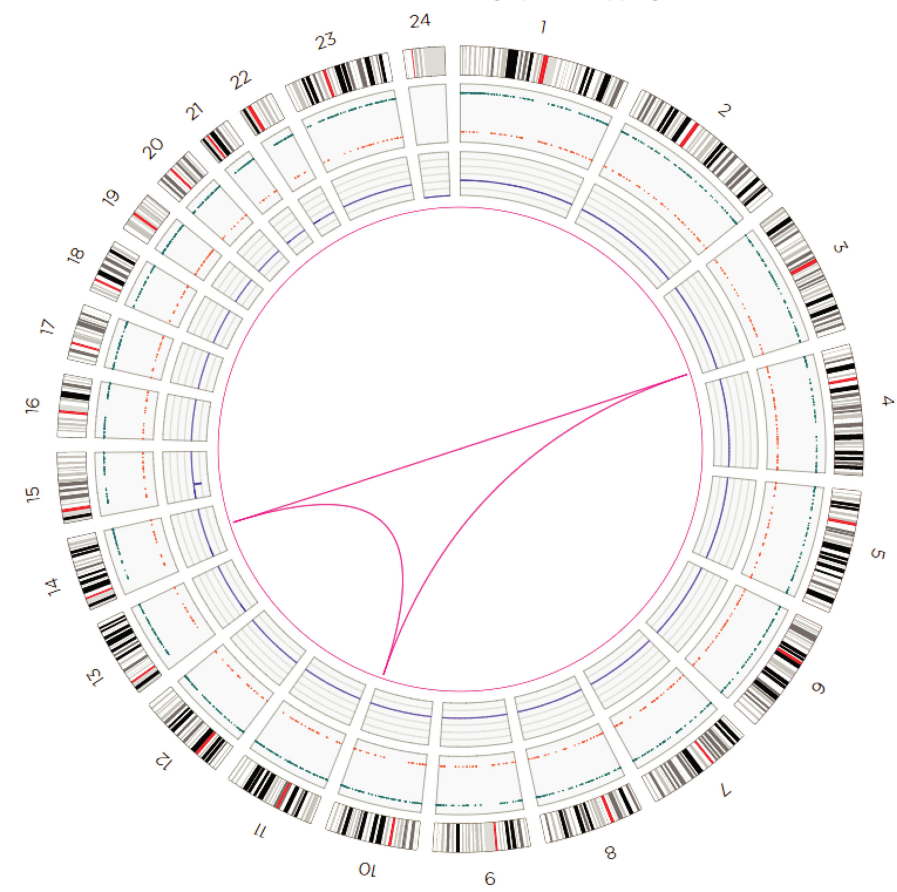

B Structural variants detected in the proband using optical mapping

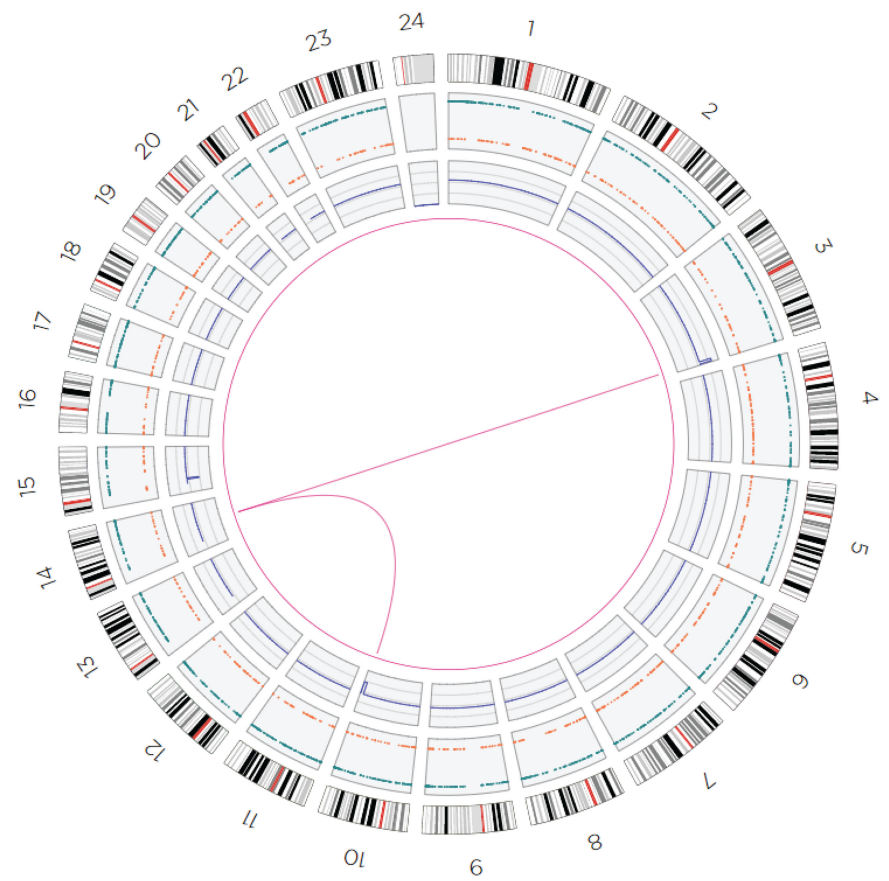

Figure 3. Circos plot illustrating structural variants detected in mother and patient using optical mapping with enzyme Nt.BspQl. The outermost bands represent the G-banding pattern of each chromosome in the human reference assembly (hg19). The next interior section shows the insertions (green dots) and deletions (orange dots) identified in the sample's assembly, and subsequently the next block displays $>2 \mathrm{Mb}$ copy-number variation (purple line) as found by rise and drop of molecule coverage. Finally, the innermost section illustrates the translocations identified. $(A)$ In the mother's sample, three translocations were identified: $t(3 ; 10), t(10 ; 14)$, and $\mathrm{t}(3 ; 14)$. A drop in coverage was observed at $\mathrm{Chr} 15$. (B) In the patient's sample, two translocations were identified: der(10)t(3;14;10) and der(14)t(3;14;10). Furthermore, a rise and drop in coverage was observed at the distal ends of Chr 3 (coinciding with the $\operatorname{der}(14) t(3 ; 14 ; 10)$ breakpoint) and Chr 10 (coinciding with the der(10) $\mathrm{t}(3 ; 14 ; 10)$ translocation breakpoint), respectively. An additional drop in coverage was observed at Chr 15. 
Often characterized by cognitive impairment, mild facial dysmorphism, and disruptive behaviors, we find similarities in phenotypic presentations between our patient and previously reported cases (Table 1). Of the 59 protein-coding genes encompassing this 8.9-Mb region, 45 are OMIM genes (Supplemental Table 1). With candidacy derived from neurodevelopmental disorders gene sets and reported gene-disease associations, 10 of these duplicated genes (Table 3) are associated with developmental disorder phenotypes (Firth et al. 2009; Guo et al. 2019). Prior literature implicates genes such as DLG1, BDH1, and PAK2 (Supplemental Table 1) in neurosynaptic development and function, suggesting they may contribute to neuropsychiatric and neurodevelopmental phenotypes (Fernández-Jaén et al. 2014; Tassano et al. 2018). Of note, the synapse-associated protein-97 encoded by DLG1 was found to have postsynaptic and presynaptic effects on synaptic transmission in primary hippocampal neurons (Rumbaugh et al. 2003), whereas TNK2 has been associated with severe cognitive regression (Supplemental Table 1). We propose $R \cup B C N$, the gene implicated in autosomal recessive spinocerebellar ataxia-15 (SCAR15; OMIM 613516), as a possible candidate gene contributing to the neurodevelopmental, behavioral, and psychiatric phenotypes observed in our patient. The main clinical features of SCAR15 include ataxic gait, dysarthria, ID, epilepsy, and delayed motor milestones. Although the duplicated nature of our mutation differs from a previous report implicating haploinsufficiency of the gene (Assoum et al. 2010), all listed features are present in our patient, with the exception of epilepsy. Future studies are needed to elucidate the distinctions between the phenotypic consequences of deletions and duplications involving the RUBCN gene.

The deleted 10q26.2qter region in our patient overlaps the Chromosome 10q26 deletion syndrome (OMIM \#609625). We previously reported four cases with distal 10q deletions (Miller et al. 2009b), and more than 100 cases of 10q26 deletion syndrome have been reported. These patients present with varying symptoms, including craniofacial anomalies, ID, urinary tract abnormalities, cardiac malformations, and neurodevelopmental and neurobehavioral conditions (Lin et al. 2016). Of the 39 protein-coding genes in the deletion region, 23 are in OMIM (Supplemental Table 2). Of the five associated with developmental disorder phenotypes (Table 3), DOCK1 and EBF3 are leading candidate causal genes. Haploinsufficiency of DOCK1 is hypothesized as the source of the phenotypic variability seen between patients with similar chromosomal abnormalities because of its involvement in the regulation and signaling of multiple pathways (Yatsenko et al. 2009). Haploinsufficiency of EBF3, a gene encoding a member of a family of highly conserved transcription factors required for central nervous system development and function (Chao et al. 2017; Tanaka et al. 2017), has been linked to neurodevelopmental disorder phenotypes such as developmental delay/ID, ataxia, hypotonia, speech impairment, strabismus, genitourinary abnormalities, mild facial dysmorphisms, and behavioral anomalies (Lopes et al. 2017; Tanaka et al. 2017). All conditions with the exception of genitourinary abnormalities were observed in our patient (Table 1). The C10orf90 gene, found within a previously suggested 600-kb minimum consensus region on 10q26.2 (Yatsenko et al. 2009), is partially deleted as it lies within our patient's $\operatorname{der}(10) t(3 ; 14 ; 10)$ breakpoint (Supplemental Fig. 2A). However, the gene is also disrupted in the patient's apparently normal mother (Supplemental Fig. 2C), suggesting it is likely not clinically significant.

The $15 q 13.2 q 13.3$ maternally inherited deletion in our patient spans a region associated with the Chromosome 15q13.3 deletion syndrome (OMIM \#612001). The highly variable phenotype can include mild to moderate ID, epilepsy, subtle dysmorphic features, behavioral problems including aggression and ADHD, and a range of other neuropsychiatric impairments (Ben-Shachar et al. 2009; Miller et al. 2009a; Shinawi et al. 2009). In some cases, the deletion may not be sufficient to cause disease (van Bon et al. 2009). In many cases $(\sim 85 \%)$, the syndrome is inherited, including from an apparently normal parent as in the case of our patient (Lowther et al. 2015). The CHRNA7 gene encoding the nicotinic alpha 7 


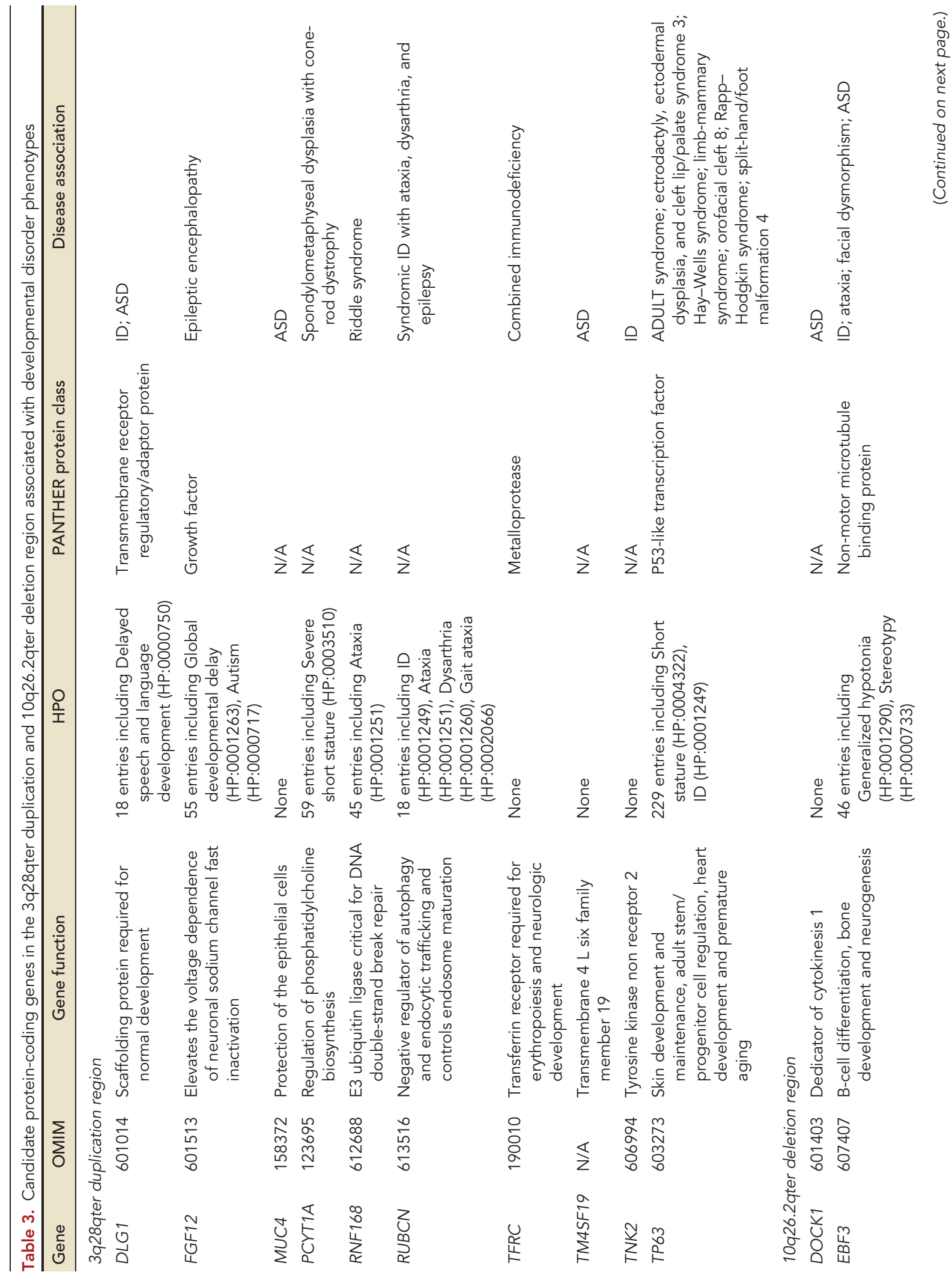




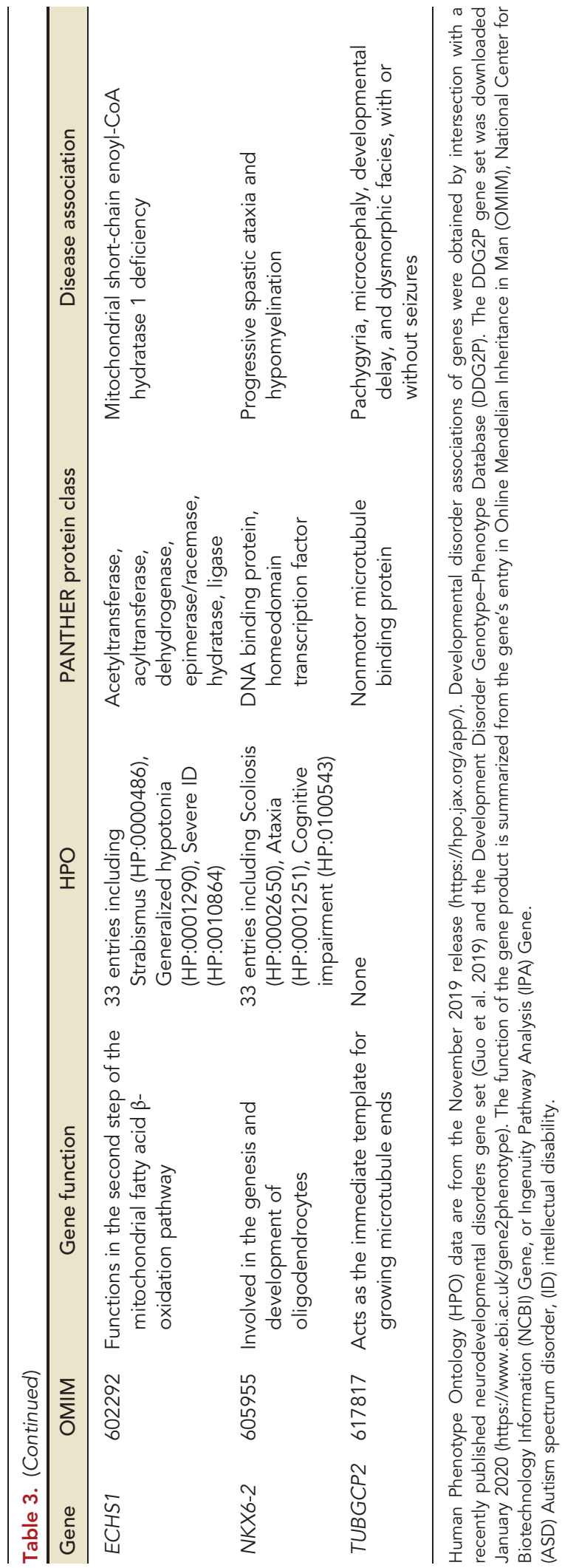


subunit cholinergic receptor has been implicated as responsible for the phenotypic spectrum seen across individuals harboring this deletion (Hoppman-Chaney et al. 2013). Although our patient's deletion was classified pathogenic in the clinical whole-genome array $\mathrm{CGH}$ report with a recommendation to test parental samples to determine if the variant is de novo or inherited, we provide a modified classification given the variant was inherited from her phenotypically normal mother (Table 2). Because of the variable expressivity, incomplete penetrance and overall unpredictability of this deletion syndrome, our patient's $15 q 13.2 q 13.3$ deletion is of uncertain significance.

The three aforementioned CNVs may contribute to the severe phenotypic presentations observed in our patient singly or in combination. Although it is difficult to map the contributions of a particular genomic variant to severe mood and behavioral conditions, given there is tremendous variability in the clinical presentation of each chromosomal insult, each case report can contribute to knowledge of the prevalence of these conditions in association with each chromosomal abnormality. Our review of the literature strongly implicates the 10q26.2qter deletion and 3q28qter duplication genes as contributory to the patient's complex phenotype, with $15 q 13.2 q 13.3$ genes possibly contributing. Phenotypes reported in patients with $10 q 26$ deletion or 3q29 duplication syndromes closely mirrored those in the patient, notably aggressive behavior with limited attention span, ID, sleep disturbance, ASD, ataxic gait, and SIB (Table 1). We also report clinical features without known association with either syndromes, as in the case of dysarthria, a condition linked to the RUBCN gene within the 3q29 region but not the 3q29 duplication syndrome. Our results highlight the utility of newer genomic technologies for improving the interpretation of complex SVs in the context of genotype-phenotype correlations.

\section{METHODS}

\section{Cytogenetics}

Prior to this study, karyotyping-based cytogenetic analysis was performed at the Genetics Centre at Guy's and St. Thomas' NHS Foundation Trust.

\section{Whole-Genome Sequencing}

DNA extracted from whole-blood samples obtained from the patient and her parents was subjected to next-generation WGS performed on an Illumina HiSeq X Ten at Macrogen Clinical Laboratories. With a targeted insert size of 300-400 bp, paired-end libraries (150 bp $\times 2$ ) were prepared using Illumina TruSeq DNA PCR-free protocol. The resulting mean depth of sequencing coverage and the corresponding mappable mean depth values are detailed in Table 4. Quality control metrics were implemented, including the

\begin{tabular}{lcc}
\hline Table 4. & Mean whole-genome sequencing coverage of reported trio & \\
\hline Sample & Mean depth of coverage & Mappable mean depth of coverage \\
\hline Patient & 45.7 & 38.9 \\
Mother & 52.4 & 44.8 \\
Father & 43.9 & 37.4 \\
\hline
\end{tabular}

${ }^{a}$ Mean depth of sequencing coverage is calculated as total yield/reference size.

${ }^{b}$ Mappable mean depth of sequencing coverage is calculated as mappable yield/reference size. 
COLD SPR ING HARBOR Molecular Case Studies
Translocations in a child with developmental delay

assessment of $\mathrm{Q} 20$ and $\mathrm{Q} 30$ bases. Sequence reads were aligned by Macrogen to the GRCh37 reference genome (hs37d5) using the Isaac aligner (version 01.15.02.08) followed by SV and CNV calling using Manta (version 0.20.2) and Control-FREEC (version 6.4), respectively.

To verify their results, we used an independent variant-calling pipeline using the Genome Analysis Toolkit (GATK) best practices (Van der Auwera et al. 2013) in a Snakemake (version 4.2.0) workflow. The workflow began by locally aligning FASTQ sequencing reads to the human reference genome (GRCh37d5) with the Burrows-Wheeler aligner (BWA-MEM; version 0.7.15), including Q15 read trimming. Duplicate reads were flagged, and split and discordant reads extracted using SAMBLASTER (version 0.1.22). The Sequence Alignment Map (SAM) files produced previously were converted to Binary Alignment Map (BAM) format and sorted using sambamba (version 0.6.1). Manta (version 0.29.6) and GenomeSTRiP (version 2.00.1665) variant callers were used to call SVs and CNVs, respectively.

\section{Whole-Genome Optical Mapping}

Optical next-generation genome mapping using the Bionano Genomics Saphyr system was used to detect SVs and CNVs in the patient and her parents. High-molecular-weight DNA (>100 kb) was prepared from whole-blood samples using Bionano Prep Blood and Cell Culture DNA Isolation Kit followed by a nick-label-repair reaction (NLR) involving (1) the nicking enzyme Nb.BssSI or Nt.BspOl, which generates single-strand nicks at its specific recognition sites (CACGAG or GCTCTTCN, respectively); (2) a DNA polymerase enzyme that incorporates fluorescent nucleotides at the nicked sites; and (3) a DNA ligase enzyme that repairs the remaining single-strand breaks. DNA was labeled using the Bionano Prep NLRS Labeling Kit (Bionano Genomics Inc.). Samples were stained with Yoyo-1 and analyzed in nanochannel array chips (Saphyr Chip, Bionano Genomics Inc.). On the chip, DNA was forced into 40-nm nanochannels using an electric field and then stretched along the channel axis for imaging. This process was carried out in automated cycles by the Saphyr instrument (Bionano Genomics Inc.). Raw images were processed and DNA molecules were detected and digitized by IrysView image processing and analysis software (Cao et al. 2014; Das et al. 2010). These molecular patterns were assembled de novo to create megabase-scale optical maps using Bionano Access (v1.0) with human haplotype-aware settings. Bionano assemblies were mapped against predicted restriction enzyme labeling sites in the human reference genome, with discrepancies between assemblies flagged as candidate sites of SV. For quality control, SVs were filtered based on quality scores of the assembly, the alignments, and the variants. The merged data sets included large assembled molecules (>150 kb) totaling $408 \mathrm{~Gb}$ and $573 \mathrm{~Gb}$ for the patient (for the Nt.BspOI and Nb.BssSl experiments, respectively), $539 \mathrm{~Gb}$ and $391 \mathrm{~Gb}$ for the mother, and $314 \mathrm{~Gb}$ and $289 \mathrm{~Gb}$ for the father. The assembly sizes were $5.74 \mathrm{~Gb}$ and $5.87 \mathrm{~Gb}$ (patient), $5.76 \mathrm{~Gb}$ and $5.89 \mathrm{~Gb}$ (mother), and $5.71 \mathrm{~Gb}$ and $5.83 \mathrm{~Gb}$ (father). Genome map N50 values ranged from 1.34 $\mathrm{Mb}$ to $4.94 \mathrm{Mb}$.

\section{Whole-Genome Array CGH}

Clinical whole-genome aCGH and SNP genotype analysis was performed by GeneDx on a custom-designed oligonucleotide microarray (GeneomeDx v5). A buccal sample from the patient was obtained from which DNA was extracted. Because of limitations of DNA quality, only copy-number aberrations of $1 \mathrm{Mb}$ or greater were reported. 


\section{ADDITIONAL INFORMATION}

\section{Data Deposition and Access}

The whole-genome sequence data for the mother/father/child trio reported in this study have been deposited in the National Data Archive (NDA) with doi:10.15154/1519367.

\section{Ethics Statement}

Written informed consent was obtained from the proband's family under a protocol approved by a Johns Hopkins School of Medicine Institutional Review Board.

\section{Acknowledgments}

We thank the family for participating in this study and permitting us to share these findings. We also thank Andy Wing Chun Pang of Bionano Genomics for help with the optical mapping analyses. We thank N. Varg for helpful discussions and comments on the manuscript.

\section{Author Contributions}

I.A.O-O., A.L.N., J.S., L.H., J.H., and J.P. wrote the manuscript. All authors critically reviewed and edited the manuscript.

\section{Funding}

Competing Interest Statement

The authors have declared no competing interest.

Received October 1, 2020; accepted in revised form November 8, 2020.
J.P. was supported by Intellectual and Developmental Disabilities Research Center (Grant U54 HD079123) from the Eunice Kennedy Shriver National Institute of Child Health and Human Development, and the support of an anonymous donor. I.A.O-O. was supported by R36 MH118005. DDG2P has been jointly funded through a MRC University Unit grant to the MRC Human Genetics Unit, the Transforming Genomic Medicine Initiative (TGMI) (Wellcome Strategic Award; grant number 200990/Z/16/Z), and the Deciphering Developmental Disorder study (Health Innovation Challenge Fund; grant number HICF1009-003).

\section{REFERENCES}

Assoum M, Salih MA, Drouot N, H'Mida-Ben Brahim D, Lagier-Tourenne C, AlDrees A, Elmalik SA, Ahmed TS, Seidahmed MZ, Kabiraj MM, et al. 2010. Rundataxin, a novel protein with RUN and diacylglycerol binding domains, is mutant in a new recessive ataxia. Brain 133: 2439-2447. doi:10.1093/brain/awq181

Ballif BC, Theisen A, Coppinger J, Gowans GC, Hersh JH, Madan-Khetarpal S, Schmidt KR, Tervo R, Escobar LF, Friedrich CA, et al. 2008. Expanding the clinical phenotype of the 3q29 microdeletion syndrome and characterization of the reciprocal microduplication. Mol Cytogenet 1: 8. doi:10.1186/1755-8166-1-8

Baptista J, Prigmore E, Gribble SM, Jacobs PA, Carter NP, Crolla JA. 2005. Molecular cytogenetic analyses of breakpoints in apparently balanced reciprocal translocations carried by phenotypically normal individuals. Eur J Hum Genet 13: 1205-1212. doi:10.1038/sj.ejhg.5201488

Barseghyan H, Tang W, Wang RT, Almalvez M, Segura E, Bramble MS, Lipson A, Douine ED, Lee H, Delot EC, et al. 2017. Next-generation mapping: a novel approach for detection of pathogenic structural variants with a potential utility in clinical diagnosis. Genome Med 9: 90. doi:10.1186/s13073-017-0479-0

Battaglia A, Novelli A, Ceccarini C, Carey JC. 2006. Familial complex 3q;10q rearrangement unraveled by subtelomeric FISH analysis. Am J Med Genet A 140A: 144-150. doi:10.1002/ajmg.a.31042

Ben-Shachar S, Lanpher B, German JR, Qasaymeh M, Potocki L, Nagamani SC, Franco LM, Malphrus A, Bottenfield GW, Spence JE, et al. 2009. Microdeletion 15q13.3: a locus with incomplete penetrance for autism, mental retardation, and psychiatric disorders. J Med Genet 46: 382-388. doi:10.1136/jmg.2008 .064378

Bullock CE, Fisher WW, Hagopian LP. 2017. Description and validation of a computerized behavioral data program: "BDataPro". Behav Anal 40: 275-285. doi:10.1007/s40614-016-0079-0 
Cao H, Hastie AR, Cao D, Lam ET, Sun Y, Huang H, Liu X, Lin L, Andrews W, Chan S, et al. 2014. Rapid detection of structural variation in a human genome using nanochannel-based genome mapping technology. Gigascience 3: 34. doi:10.1186/2047-217X-3-34

Chao HT, Davids M, Burke E, Pappas JG, Rosenfeld JA, McCarty AJ, Davis T, Wolfe L, Toro C, Tifft C, et al. 2017. A syndromic neurodevelopmental disorder caused by de novo variants in EBF3. Am J Hum Genet 100: 128-137. doi:10.1016/j.ajhg.2016.11.018

Courtens W, Wuyts W, Rooms L, Pera SB, Wauters J. 2006. A subterminal deletion of the long arm of chromosome 10: a clinical report and review. Am J Med Genet A 140A: 402-409. doi:10.1002/ajmg.a.31053

Das SK, Austin MD, Akana MC, Deshpande P, Cao H, Xiao M. 2010. Single molecule linear analysis of DNA in nano-channel labeled with sequence specific fluorescent probes. Nucleic Acids Res 38: e177. doi:10.1093/ nar/gkq673

Fernández-Jaén A, Castellanos MDC, Fernández-Perrone AL, Fernández-Mayoralas DM, de la Vega AG, Calleja-Pérez B, Fernández EC, Albert J, Hombre MCS. 2014. Cerebral palsy, epilepsy, and severe intellectual disability in a patient with 3q29 microduplication syndrome. Am J Med Genet A 164: 2043-2047. doi:10.1002/ajmg.a.36559

Firth HV, Richards SM, Bevan AP, Clayton S, Corpas M, Rajan D, Van Vooren S, Moreau Y, Pettett RM, Carter NP. 2009. DECIPHER: database of chromosomal imbalance and phenotype in humans using ensembl resources. Am J Hum Genet 84: 524-533. doi:10.1016/j.ajhg.2009.03.010

Guo H, Duyzend MH, Coe BP, Baker C, Hoekzema K, Gerdts J, Turner TN, Zody MC, Beighley JS, Murali SC, et al. 2019. Genome sequencing identifies multiple deleterious variants in autism patients with more severe phenotypes. Genet Med 21: 1611-1620. doi:10.1038/s41436-018-0380-2

Hagopian LP, Fisher WW, Thompson RH, Owen-DeSchryver J, Iwata BA, Wacker DP. 1997. Toward the development of structured criteria for interpretation of functional analysis data. J Appl Behav Anal 30: 313-325. doi:10.1901/jaba.1997.30-313

Hoppman-Chaney N, Wain K, Seger PR, Superneau DW, Hodge JC. 2013. Identification of single gene deletions at 15q13.3: further evidence that CHRNA7 causes the 15q13.3 microdeletion syndrome phenotype. Clin Genet 83: 345-351. doi:10.1111/j.1399-0004.2012.01925.x

Lawrence MB, Arreola A, Cools M, Elton S, Wood KS. 2017. 3q29 Chromosomal duplication in a neonate with associated myelomeningocele and midline cranial defects. Clin Dysmorphol 26: 221-223. doi:10.1097/ MCD. 0000000000000193

Lin S, Zhou Y, Fang Q, Wu J, Zhang Z, Ji Y, Luo Y. 2016. Chromosome 10q26 deletion syndrome: two new cases and a review of the literature. Mol Med Rep 14: 5134-5140. doi:10.3892/mmr.2016.5864

Lisi EC, Hamosh A, Doheny KF, Squibb E, Jackson B, Galczynski R, Thomas GH, Batista DAS. 2008. 3q29 interstitial microduplication: a new syndrome in a three-generation family. Am J Med Genet A 146A: 601609. doi:10.1002/ajmg.a.32190

Lopes F, Soares G, Gonçalves-Rocha M, Pinto-Basto J, Maciel P. 2017. Whole gene deletion of EBF3 supporting haploinsufficiency of this gene as a mechanism of neurodevelopmental disease. Front Genet 8: 143 doi:10.3389/fgene.2017.00143

Lowther C, Costain G, Stavropoulos DJ, Melvin R, Silversides CK, Andrade DM, So J, Faghfoury H, Lionel AC, Marshall CR, et al. 2015. Delineating the 15q13.3 microdeletion phenotype: a case series and comprehensive review of the literature. Genet Med 17: 149-157. doi:10.1038/gim.2014.83

Miller DT, Shen Y, Weiss LA, Korn J, Anselm I, Bridgemohan C, Cox GF, Dickinson H, Gentile J, Harris DJ, et al. 2009a. Microdeletion/duplication at 15q13.2q13.3 among individuals with features of autism and other neuropsychiatric disorders. J Med Genet 46: 242-248. doi:10.1136/jmg.2008.059907

Miller ND, Nance MA, Wohler ES, Hoover-Fong JE, Lisi E, Thomas GH, Pevsner J. 2009b. Molecular (SNP) analyses of overlapping hemizygous deletions of $10 \mathrm{q} 25.3$ to $10 \mathrm{qter}$ in four patients: evidence for HMX2 and HMX3 as candidate genes in hearing and vestibular function. Am J Med Genet A 149A: 669-680. doi:10.1002/ajmg.a.32705

Ordulu Z, Kammin T, Brand H, Pillalamarri V, Redin CE, Collins RL, Blumenthal I, Hanscom C, Pereira S, Bradley I, et al. 2016. Structural chromosomal rearrangements require nucleotide-level resolution: lessons from next-generation sequencing in prenatal diagnosis. Am J Hum Genet 99: 1015-1033. doi:10.1016/j.ajhg .2016 .08 .022

Pollak RM, Zinsmeister MC, Murphy MM, Zwick ME, Mulle JG. 2020. New phenotypes associated with 3q29 duplication syndrome: results from the 3q29 registry. Am J Med Genet A 182: 1152-1166. doi:10.1002/ ajmg.a.61540

Rumbaugh G, Sia GM, Garner CC, Huganir RL. 2003. Synapse-associated protein-97 isoform-specific regulation of surface AMPA receptors and synaptic function in cultured neurons. J Neurosci 23: 4567-4576. doi:10.1523/JNEUROSCI.23-11-04567.2003

Shinawi M, Schaaf CP, Bhatt SS, Xia Z, Patel A, Cheung SW, Lanpher B, Nagl S, Herding HS, Nevinny-Stickel C, et al. 2009. A small recurrent deletion within $15 q 13.3$ is associated with a range of neurodevelopmental phenotypes. Nat Genet 41: 1269-1271. doi:10.1038/ng.481 
Sparrow SS, Cicchetti DV, Balla DA. 2005. Vineland adaptive behavior scales, 2nd ed. American Guidance Service, Circle Pines, MN.

Talkowski ME, Rosenfeld JA, Blumenthal I, Pillalamarri V, Chiang C, Heilbut A, Ernst C, Hanscom C, Rossin E, Lindgren AM, et al. 2012. Sequencing chromosomal abnormalities reveals neurodevelopmental loci that confer risk across diagnostic boundaries. Cell 149: 525-537. doi:10.1016/j.cell.2012.03.028

Tanaka AJ, Cho MT, Willaert R, Retterer K, Zarate YA, Bosanko K, Stefans V, Oishi K, Williamson A, Wilson GN, et al. 2017. De novo variants in EBF3 are associated with hypotonia, developmental delay, intellectual disability, and autism. Cold Spring Harb Mol Case Stud 3: a002097. doi:10.1101/mcs.a002097

Tassano E, Uccella S, Giacomini T, Severino M, Siri L, Gherzi M, Celle ME, Porta S, Gimelli G, Ronchetto P. 2018. 3q29 microduplication syndrome: description of two new cases and delineation of the minimal critical region. Eur J Med Genet 61: 428-433. doi:10.1016/j.ejmg.2018.02.011

van Bon BW, Mefford HC, Menten B, Koolen DA, Sharp AJ, Nillesen WM, Innis JW, de Ravel TJ, Mercer CL, Fichera $\mathrm{M}$, et al. 2009. Further delineation of the 15q13 microdeletion and duplication syndromes: a clinical spectrum varying from non-pathogenic to a severe outcome. J Med Genet 46: 511-523. doi:10.1136/jmg .2008 .063412

Van der Auwera GA, Carneiro MO, Hartl C, Poplin R, del Angel G, Levy-Moonshine A, Jordan T, Shakir K, Roazen D, Thibault J, et al. 2013. From FastQ data to high confidence variant calls: the Genome Analysis Toolkit best practices pipeline. Curr Protoc Bioinformatics 43: 11 10 11-11 1033.

Vassos E, Collier DA, Holden S, Patch C, Rujescu D, St Clair D, Lewis CM. 2010. Penetrance for copy number variants associated with schizophrenia. Hum Mol Genet 19: 3477-3481. doi:10.1093/hmg/ddq259

Weckselblatt B, Hermetz KE, Rudd MK. 2015. Unbalanced translocations arise from diverse mutational mechanisms including chromothripsis. Genome Res 25: 937-947. doi:10.1101/gr.191247.115

Yatsenko S, Kruer M, Bader P, Corzo D, Schuette J, Keegan C, Nowakowska B, Peacock S, Cai W, Peiffer D, et al. 2009. Identification of critical regions for clinical features of distal $10 \mathrm{q}$ deletion syndrome. Clin Genet 76: 54-62. doi:10.1111/j.1399-0004.2008.01115.x 


\section{COLD SPRING HARBOR Molecular Case Studies}

\section{Characterization of an unbalanced translocation causing 3q28qter duplication and 10q26.2qter deletion in a patient with global developmental delay and self-injury}

Ikeoluwa A. Osei-Owusu, Alexis L. Norris, Anya T. Joynt, et al.

Cold Spring Harb Mol Case Stud 2020, 6: a005884

Access the most recent version at doi: $10.1101 / \mathrm{mcs} . a 005884$

Supplementary
Material $\quad \underset{C 1}{\text { htt://molecularcasestudies.cshlp.org/content/suppl/2020/12/17/mcs.a005884.D }}$

References This article cites 35 articles, 6 of which can be accessed free at:

http://molecularcasestudies.cshlp.org/content/6/6/a005884.full.html\#ref-list-1

License This article is distributed under the terms of the Creative Commons

Attribution-NonCommercial License, which permits reuse and redistribution, except for commercial purposes, provided that the original author and source are credited.

Email Alerting Receive free email alerts when new articles cite this article - sign up in the box at the Service top right corner of the article or click here. 